\title{
Erratum to: The need for differentiating diabetes-specific mortality from total mortality when comparing metformin with insulin regarding cancer survival
}

\author{
Min Wei ${ }^{1,3} \cdot$ Yu Liu ${ }^{2}$ Chunhong Wang ${ }^{3}$ Chuanhua $\mathrm{Yu}^{3} \cdot$ Dejia $\mathbf{L i}^{3}$. \\ Wei Zhou $^{3} \cdot$ Zhi-Jiang Zhang ${ }^{3}$
}

Published online: 19 December 2016

(C) Springer-Verlag Italia 2016

\section{Erratum to: Acta Diabetol \\ DOI 10.1007/s00592-016-0933-2}

The author would like to add a word "Natural" in the Acknowledgements section. It should read as: This work was supported by the National Natural Science Foundation of China (Grant 81641123).

And the word "such" is added to the last sentence in the article. It should read as: Metformin's potential antitumor effect is such an example.

The online version of the original article can be found under doi:10.1007/s00592-016-0933-2.

Yu Liu

1yu429@163.com

1 Department of Obstetrics and Gynecology, Renmin Hospital of Wuhan University, Wuhan University, Wuhan, China

2 Department of Statistics and Public Administration, College of Management, Wuhan Institute of Technology, 206 Optical Valley Road, Wuhan, China

3 Department of Epidemiology and Biostatistics, School of Public Health, Wuhan University, Wuhan, China 